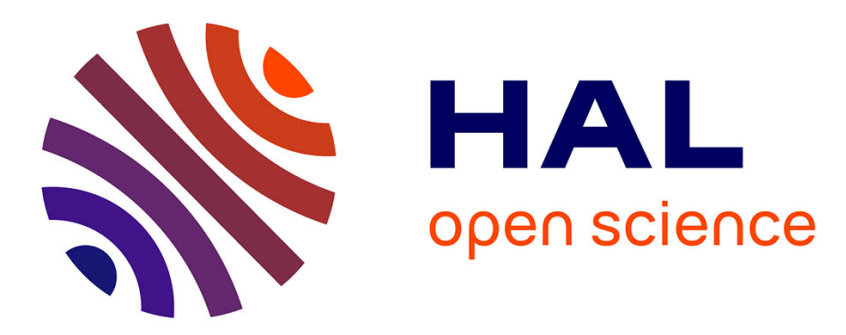

\title{
Inscribing diffraction gratings in bulk silicon with nanosecond laser pulses
}

\author{
M. Chambonneau, D. Richter, S. Nolte, D. Grojo
}

\section{To cite this version:}

M. Chambonneau, D. Richter, S. Nolte, D. Grojo. Inscribing diffraction gratings in bulk silicon with nanosecond laser pulses. Optics Letters, 2018, 43 (24), pp.6069. 10.1364/OL.43.006069 . hal02106517

\section{HAL Id: hal-02106517 https://hal-amu.archives-ouvertes.fr/hal-02106517}

Submitted on 23 Apr 2019

HAL is a multi-disciplinary open access archive for the deposit and dissemination of scientific research documents, whether they are published or not. The documents may come from teaching and research institutions in France or abroad, or from public or private research centers.
L'archive ouverte pluridisciplinaire HAL, est destinée au dépôt et à la diffusion de documents scientifiques de niveau recherche, publiés ou non, émanant des établissements d'enseignement et de recherche français ou étrangers, des laboratoires publics ou privés. 


\title{
Inscribing diffraction gratings in bulk silicon with nanosecond laser pulses
}

\author{
M. Chambonneau, ${ }^{1,2, *}$ (1) D. Richter, ${ }^{2}$ S. Nolte, ${ }^{2,3}$ and D. Grojo ${ }^{1}$ \\ ${ }^{1}$ Aix-Marseille University, CNRS, LP3, F-13288 Marseille, France \\ ${ }^{2}$ Institute of Applied Physics, Abbe Center of Photonics, Friedrich Schiller University Jena, Albert-Einstein-Straße 15, 07745 Jena, Germany \\ ${ }^{3}$ Fraunhofer Institute for Applied Optics and Precision Engineering, Albert-Einstein-Straße 7, 07745 Jena, Germany \\ *Corresponding author: maxime.chambonneau@uni-jena.de
}

Received 19 October 2018; revised 13 November 2018; accepted 14 November 2018; posted 15 November 2018 (Doc. ID 348711); published 13 December 2018

\begin{abstract}
Diffraction gratings are transversally inscribed in the bulk of monolithic crystalline silicon with infrared nanosecond laser pulses. Nanoscale material analyses of the modifications composing the gratings show that they rely on laserinduced stress associated with a positive refractive index change as confirmed with phase-shift interferometry. Characterizations of the optical properties of the gratings, including the diffraction angles and the efficiency of the different orders, are carried out. The refractive index change obtained from these measurements is in good agreement with the phase-shift measurements. Finally, we show that the grating diffraction efficiency depends strongly on the laser writing speed. (c) 2018 Optical Society of America
\end{abstract}

https://doi.org/10.1364/OL.43.006069

Ultrafast laser pulses (i.e., in the femtosecond regime) offer the possibility to tailor the properties of glasses for writing waveguides [1], storing data eternally [2], and inducing secondharmonic generation [3], for instance. By inscribing permanent diffraction gratings in the bulk of fused silica, Sudrie et al. have demonstrated that the control of the refractive index is essential for the functionalization of the material [4]. Transposing this technique to crystalline silicon (c-Si) would be of broad interest for applications in silicon photonics, terahertz physics, and Raman silicon lasers [5]. Moreover, the laser inscription of diffraction gratings in this semiconductor material would open the path to the writing of Bragg gratings, as it has existed in glasses for decades [6]. However, the main issue associated with the interaction between infrared (IR) femtosecond laser pulses and the bulk c-Si through a plane surface sample is the delocalization of the energy mainly due to plasma defocusing [7]. To date, three strategies have been adopted for overcoming these limitations and functionalizing c-Si. The first one relies on femtosecond irradiation through a spherical interface for completely suppressing the refraction at the surface [8]. The second strategy consists of a femtosecond irradiation at a high repetition rate $(250 \mathrm{kHz})$ provoking cumulative effects [9]. Finally, the much less costly third approach employs pulses with a duration on the order of or higher than 1 ps for drastically reducing the nonlinear and plasma effects protecting the bulk of $\mathrm{c}-\mathrm{Si}[10-12]$. The underlying mechanisms are the production of a dense plasma induced by two-photon absorption followed by high-temperature hydrodynamic phenomena. Indeed, analyses of the laser-induced modifications have revealed that these pulses are able to produce a wide variety of material structures from voids to densified phases [13-16]. Therefore, this emerging long pulse duration regime could be suitable for functionalizing c-Si in the volume. Recently, Tokel $e t a l$. have suggested the possibility to inscribe gratings based on a negative refractive index change inside silicon for evaluating the efficiency of holograms written by back-reflected nanosecond laser pulses [11]. However, no characterizations of such components were provided.

In this Letter, we demonstrate the inscription of diffraction gratings in the bulk of c-Si with nanosecond pulses at $0.01 \mathrm{~mm} / \mathrm{s}$ writing speed and analyze their optical properties. Material characterizations of the laser-written lines composing the gratings show that the modifications consist of stress, as confirmed by quantitative phase-shift interferometry which, moreover, gives access to a refractive index change of $3.6 \times 10^{-3}$. The injection of IR continuous-wave (CW) light in the gratings enables us to evaluate their optical properties in terms of angles and diffraction efficiency of the different orders. Moreover, the measurements of the grating geometry, as well as the diffraction efficiency, allow us to retrieve a theoretical value for the refractive index change which is consistent with the one found by phase-shift measurements. Finally, gratings inscribed at $0.5 \mathrm{~mm} / \mathrm{s}$ exhibit a mediocre diffraction efficiency, which is explained by the morphology of the written lines and confirms that the adjustment of the writing speed is paramount for optimizing the function given to the material.

The experimental setup employed for writing diffraction gratings inside c-Si is schematically depicted in Fig. 1. It relies on 5 ns duration (full width at half-maximum) Gaussian laser pulses (in red) at a $1.55 \mu \mathrm{m}$ wavelength and a $1 \mathrm{kHz}$ repetition rate emitted by an Er-doped fiber laser (MWTech, PFL-1550). The beam is focused inside a $1 \mathrm{~mm}$ thick (100)-oriented monolithic c-Si sample by means of an objective lens of numerical aperture NA $=0.7$. At the focus positioned at the center of the sample, the beam is Gaussian with a waist $w_{0}=1.4 \mu \mathrm{m}$ 


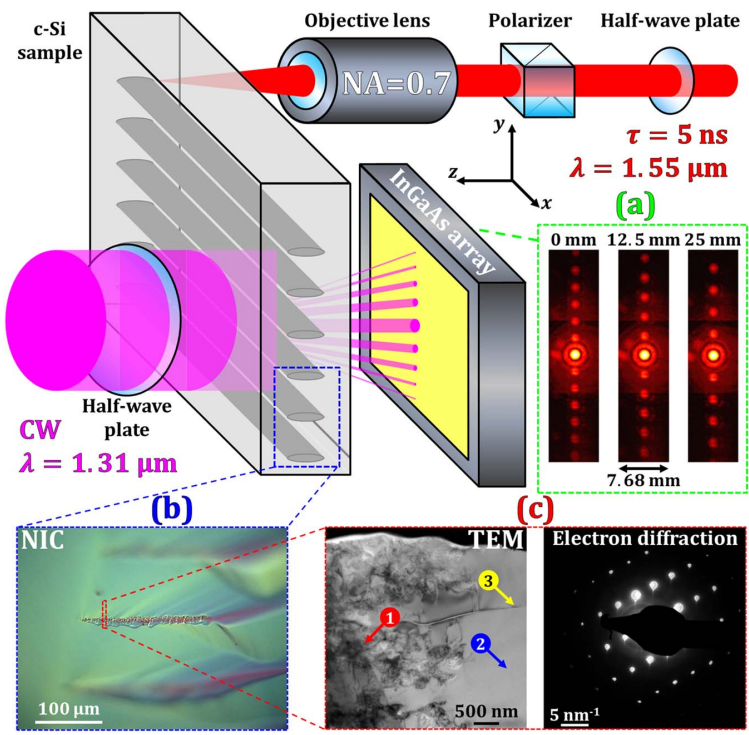

Fig. 1. Schematic description of the experimental arrangement. The red and pink beams correspond to the one employed for nanosecond writing and for analyzing the optical properties of the inscribed gratings, respectively. (a) Diffraction patterns recorded at the indicated relative $z$ positions of the InGaAs array. (b) NIC micrograph of the cross section of a grating. (c) TEM image, including the material (1) modified and (2) unmodified by the laser, as well as (3) a cleavinginduced crack and electron diffraction image taken in (1).

measured with an InGaAs camera (Raptor, OWL SWIR 640) and a microscope based on an objective lens of $\mathrm{NA}=0.85$. The maximum laser pulse energy measured in air before the sample is adjusted by means of a half-wave plate combined with a polarizer. It is kept at $5.3 \mu \mathrm{J}$, corresponding to a maximum fluence of $86 \mathrm{~J} / \mathrm{cm}^{2}$. The gratings consisting of 20 and 41 parallel lines separated with a period $\Lambda=100$ and $50 \mu \mathrm{m}$, respectively, are written transversally by moving the sample along the $x$ axis at $0.01 \mathrm{~mm} / \mathrm{s}$. For characterizing the optical properties of the gratings, CW light emitted at a $1.31 \mu \mathrm{m}$ (in pink) wavelength by a superluminescent diode (Thorlabs, SLD1018S) illuminates the periodic structures with controlled polarization, thanks to a half-wave plate. The diffraction pattern is analyzed post-mortem by means of the aforementioned InGaAs camera mounted on stages allowing its displacement in the $z$ direction for evaluating the angle of diffraction for the different orders, as well as in the $(x, y)$ plane for image stitching. In Fig. 1(a), three examples of recorded diffraction patterns with steps of $12.5 \mathrm{~mm}$ along the $z$ axis are displayed. Material characterizations have been performed after cleaving the sample along the $y$ axis. First, the Nomarski interference contrast (NIC) image displayed in Fig. 1(b) gives us access to the geometry of the modification. The weighted average width of the lines is $\sim 4.4 \mu \mathrm{m}$, and the length is $\sim 187 \mu \mathrm{m}$ along the $z$ axis. Secondly, transmission electron microscopy (TEM), as well as the electron diffraction images in Fig. 1(c), have been obtained, thanks to the preparation of a thin lamella ( $\sim 100 \mathrm{~nm}$ thickness) with a gallium double-beam focused ion beam (TESCAN, LYRA3). Although the modification containing randomly distributed features is visible in TEM, the electron diffraction image acquired with an electron beam of a few hundreds of nanometers in diameter reveals that the material is essentially mono-crystalline with a diffraction pattern and, thus, a crystalline structure, identical to the unmodified material. Similar electron diffraction patterns have been obtained at different points of the laser-modified zone. These results lead to the conclusion that the written lines are mainly based on locally induced stress inside the material. Ultimately, the refractive index of the lines composing the gratings is measured along the $z$ axis by means of the phase-shift microscope based on IR Mach-Zehnder interferometry (not shown in Fig. 1) detailed in [14]. For cost and compactness considerations, the femtosecond laser source used in the originally developed instrument was replaced by the aforementioned superluminescent diode exhibiting a short coherence length so that the parasitic interferences caused by multiple reflections can be as efficiently avoided.

A typical phase-shift measurement is displayed in Fig. 2. Similar to Ref. [8], two points have been defined for obtaining these traces. The first one (red data) is centered on a line composing a grating, and the second one is a reference point (blue data) located on the same iso-phase curve in an area unmodified by the nanosecond beam. Both of them are observed in the $(x, y)$ plane. One can note the relative decrease in the maximum transmitted intensity of the object compared to the reference due to absorption. The optical path difference $\delta$ between the two arms of the interferometer is adjusted with nanometer precision, thanks to a piezoelectric stage. As highlighted by the inset, the distance $\delta$ between two maxima is the wavelength $\lambda=1.31 \mu \mathrm{m}$ in accordance with theory. The data have been fitted by multiplying a Gaussian envelope with the term $\sin \left(2 \pi \delta / \lambda+\varphi_{0}+\Delta \varphi\right)$, where $\varphi_{0}$ is the initial phase, and $\Delta \varphi$ is the phase shift between the considered signal and the reference (i.e., it is zero for fitting the reference data). The phase shift between the two signals in Fig. 2 is measured to be $\Delta \varphi=3.25 \mathrm{rad}$. The positive sign of this value was additionally confirmed (1) by comparing the relative positions of the occasional jumps in the trace amplitude on a few measurements due to instabilities, and (2) by four-step measurements similar to those in Ref. [14]. According to the length $d=187 \mu \mathrm{m}$ of the line extracted from the NIC image in Fig. 1, this phase shift corresponds to a refractive index change $\Delta n=\lambda \Delta \varphi / 2 \pi d=3.6 \times 10^{-3}$.

The first property of the inscribed gratings investigated is the angle of diffraction of the different orders $m$. The theoretical value of these angles can be obtained with the grating

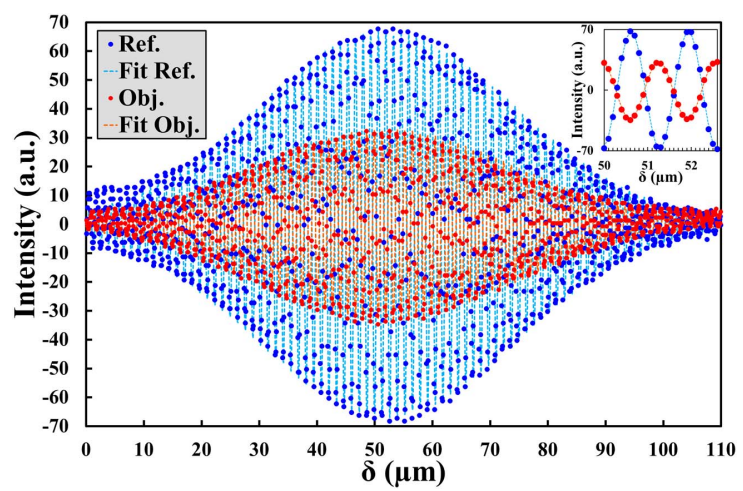

Fig. 2. Interference traces obtained on the same iso-phase curve in a reference point unmodified by the nanosecond laser beam during the grating inscription (in blue) and one in the center of a line composing the grating (in red). The inset is a magnification at the center of the traces. 
equation [17] for an irradiation at normal incidence $n_{\mathrm{Si}} \sin \left(\theta_{\mathrm{Si}}\right)=m \lambda / \Lambda$, where $n_{\mathrm{Si}}=3.5$ is the refractive index of $\mathrm{c}-\mathrm{Si}$ at $\lambda=1.31 \mu \mathrm{m}$ wavelength, $\theta_{\mathrm{Si}}$ is the angle for the $m$ th order of diffraction in silicon, and $\Lambda$ is the grating period. In order to obtain angles $\theta_{\text {air }}$ comparable to the measurements performed in air (refractive index $\sim 1$ ), Snell's law has been applied, yielding

$$
\theta_{\text {air }}=\arcsin (m \lambda / \Lambda)
$$

The calculated values of $\theta_{\text {air }}$ are reported in Fig. 3 for the different orders of diffraction $m$ for two gratings with a period $\Lambda=$ 100 and $50 \mu \mathrm{m}$, respectively. As expected, for an identical order $m$, the shorter the period $\Lambda$, the higher the $\theta_{\text {air }}$ values. These theoretical values are compared with the ones experimentally measured. Excellent agreement is found between the theoretical and experimental values, showing the precision of the inscription process.

The second optical property of the written gratings determined is the diffraction efficiency for various polarizations. Figure 4(a) shows diffraction patterns normalized to the 0th order, where a perpendicular (left) and a parallel (right) polarization with respect to the written lines have been employed for gratings with a period $\Lambda=50$ (top) and $100 \mu \mathrm{m}$ (bottom), respectively. Our previous conclusions about the angle of diffraction drawn from Fig. 3 are retrieved in Fig. 4(a). Moreover, the diffraction efficiency is much higher for a polarization perpendicular to the lines in comparison with the case where the electric field is parallel to them. This result, which is similar to gratings written in the bulk of fused silica [4], is more extensively studied in Fig. 4(b) for the grating of period $\Lambda=50$ $\mu \mathrm{m}$. In the optimal configuration where the polarization is $90^{\circ}$, the ratio between the 0 th and the 1 st order is $\sim 10.4 \%$, while it is $\sim 5.1 \%$ when the polarization is $0^{\circ}$. Although this maximum diffraction efficiency is lower than the one for gratings in fused silica, this proof of concept makes a promising step toward the inscription of optical components in the bulk of c-Si which could be improved in the future by applying successive scans [4]. Moreover, the inscription of gratings offers a complementary measurement of the refractive index accessible by laser writing with simulation of the propagation of the light across the periodic structure. The theoretical values for the diffraction efficiency are shown in Fig. 4(b) (empty red squares). They have been calculated by applying the rigorous coupled-wave

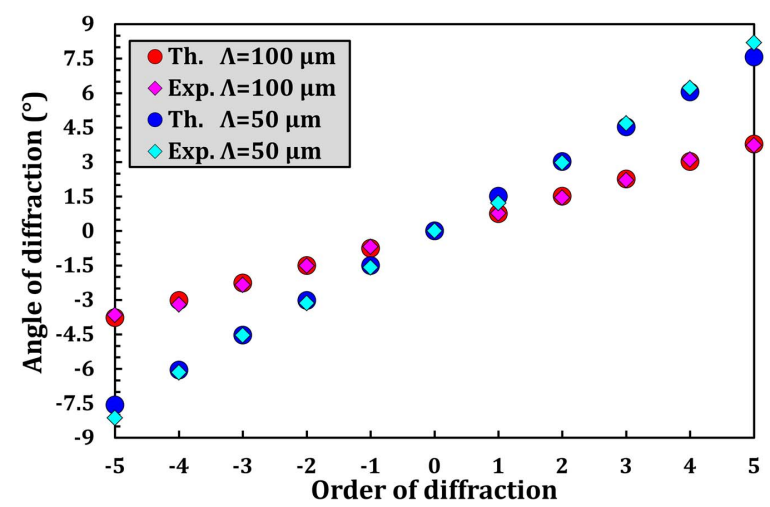

Fig. 3. Comparison between the theoretical values (circles) of the angle of diffraction for the different orders according to Eq. (1) and experimental measurements (squares) for gratings of period $\Lambda=100$ (red) and $50 \mu \mathrm{m}$ (blue).

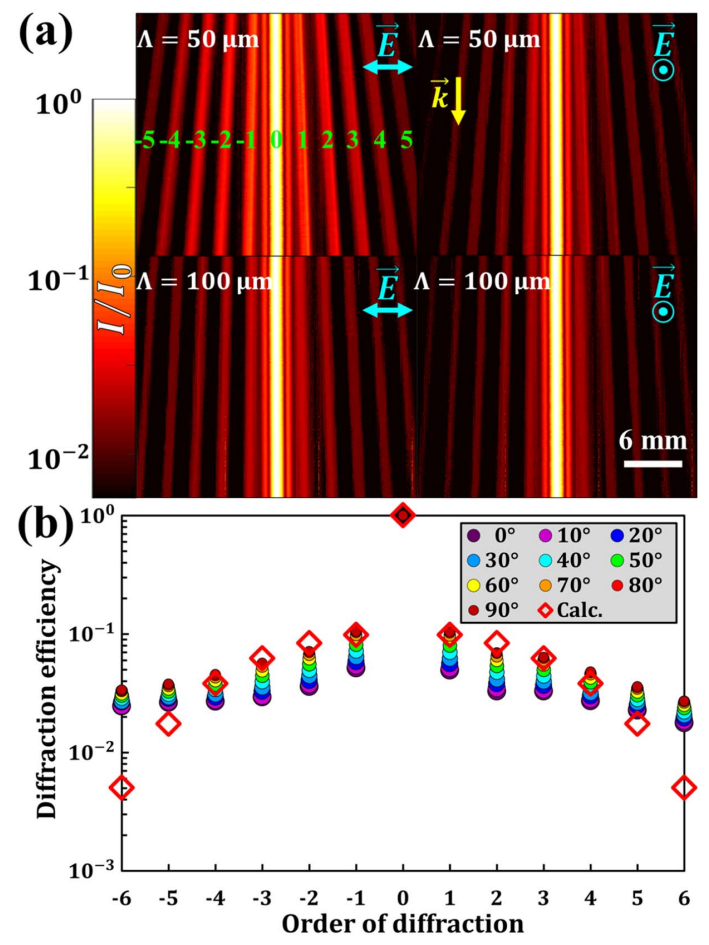

Fig. 4. (a) Diffraction patterns normalized to the 0 th order obtained on gratings with indicated periods $\Lambda$ for an incident polarization indicated by the vector $\vec{E}$. The orders of diffraction are indicated in green. The vector $\vec{k}$ shows the direction of propagation. The spatial scale applies to all images. (b) Diffraction efficiencies for a grating of period $\Lambda=50 \mu \mathrm{m}$, at indicated polarizations. $0^{\circ}$ and $90^{\circ}$ stand for a polarization parallel and perpendicular to the lines, respectively. The circles are measurements, and the empty red squares are calculations at $90^{\circ}$ according to Ref. [18].

analysis [18], assuming that the lines have a rectangular cross section with a width of $4.4 \mu \mathrm{m}$ and a length of $187 \mu \mathrm{m}$ in the $y$ and $z$ directions, respectively. By only adjusting the $\Delta n$ value homogeneously distributed along the lines, the calculated diffraction efficiency of the different orders fits the experimental measurements. While very good agreement between calculations and measurements is found for the first four orders, one can note some deviations to the theory for the 5th and the 6th orders likely due to the limited dynamics of the employed camera. This best agreement shown here is obtained for a theoretical refractive index change value of $3.0 \times 10^{-3}$, which is very close to the one obtained in Fig. 2, where $\Delta n=3.6 \times 10^{-3}$. The difference of $\sim 17 \%$ between both estimates can be reasonably attributed to the assumptions made for simulations, namely the homogeneous distribution of $\Delta n$ and the rectangular cross section, as well as the accuracy of the phase-shift measurements of $\pm 10 \%$. Additionally, the simulations reveal that the diffraction efficiency should be independent of the incident polarization. This is due to the high period-to-wavelength ratio, as well as the low optical path difference between the lines and the unmodified material, implying that this problem can also be treated in a scalar and paraxial way combined with the thin element approach [19]. Thereby, the polarization-dependent experimental results in Fig. 4 shed light on the stress-induced birefringent nature of the modifications, in excellent agreement with the nanoscale characterizations in Fig. 1(c). 


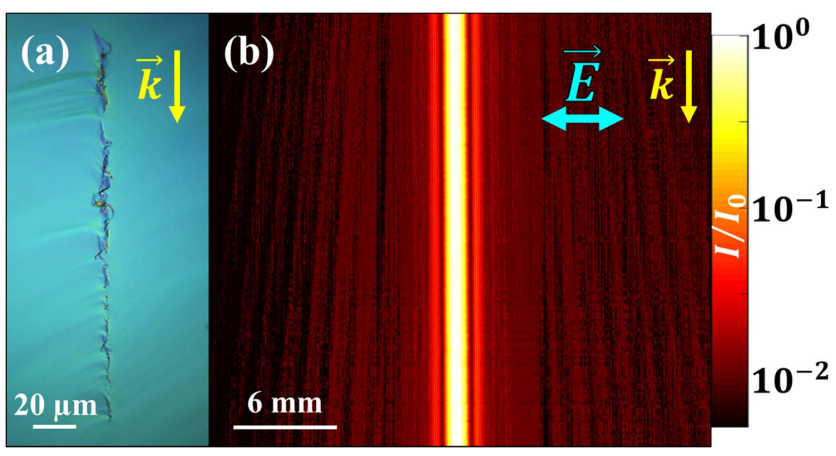

Fig. 5. (a) NIC micrograph of a cross section of one line composing a grating of period $\Lambda=100 \mu \mathrm{m}$ written at $0.5 \mathrm{~mm} / \mathrm{s}$. (b) Diffraction pattern of the corresponding grating. The vector $\vec{k}$ shows the direction of propagation.

Ultimately, in order to demonstrate possibilities to tailor the optical properties of silicon, another grating with a period $\Lambda=$ $100 \mu \mathrm{m}$ has been written at $0.5 \mathrm{~mm} / \mathrm{s}$. The cross section of one of the lines composing the grating is shown in Fig. 5(a). At this high speed, similar to Ref. [10], the morphology takes the form of non-homogeneously distributed cracks. By carrying out phase measurements similar to those in Fig. 2, a negative phase shift of $\Delta \varphi=-3.6 \mathrm{rad}$ is retrieved, confirming that this morphology is likely composed of voids [15]. The repetition rate of $1 \mathrm{kHz}$ (ensuring no heat accumulation on a pulse-to-pulse basis) and beam diameter at $1 / \mathrm{e}^{2}$ of $2 w_{0}=2.8 \mu \mathrm{m}$ implies that the number of pulses per point of the grating is 280 and 5 at the writing speed of 0.01 and $0.5 \mathrm{~mm} / \mathrm{s}$, respectively. Therefore, the material changes from pulse to pulse play a major role in the inscription process. Because of the crack morphology exhibited in Fig. 5(a), we assume that the positive refractive index change obtained at $0.01 \mathrm{~mm} / \mathrm{s}$ is produced by the motion of $\mathrm{c}-\mathrm{Si}$ which is melted by each pulse and fills the voids and the cracks left by the preceding ones. Although the line in Fig. 5(a) has approximately the same length along the $z$ axis as the one in Fig. $1(\sim 190 \mu \mathrm{m})$, the width is much lower $(\sim 2 \mu \mathrm{m})$. Consequently, the diffraction efficiency shown in Fig. 5(b) is poor, compared to the one in the bottom left of Fig. 4(a) for the two gratings with an identical period illuminated under the same conditions. Indeed, in Fig. 5(b), the orders of diffraction are not well defined, and their intensity is very low compared to the 0 th order. Therefore, we conclude that the efficiency of the gratings inscribed inside silicon with nanosecond pulses is extremely dependent on the writing speed.

To summarize, diffraction gratings have been inscribed transversally in the bulk of c-Si with tightly focused IR nanosecond pulses. Post-mortem characterizations of the lines written at $0.01 \mathrm{~mm} / \mathrm{s}$ reveal that the laser-induced modifications consist of stress inside the material which mainly remains monocrystalline without the presence of voids. This feature is confirmed by phase-shift microscopy which enables us to estimate the average refractive index change $\Delta n=3.6 \times 10^{-3}$. The optical properties of the inscribed gratings have been determined in terms of angles of diffraction and efficiency, both in good agreement with theory. Finally, gratings written at higher speeds are composed of lines exhibiting a crack morphology, and their efficiency is drastically lowered compared to the ones inscribed at $0.01 \mathrm{~mm} /$ s. To go further in the comprehension, the refractive index change, as well as the grating efficiency, will be evaluated after thermal annealing processes at various temperatures.

Funding. H2020 European Research Council (ERC) (724480); Conseil Régional Provence-Alpes-Côte d'Azur (APEX2016 IR-SCOPE); NUCLEUS, Bundesministerium für Bildung und Forschung (BMBF) (03IHS107A).

\section{REFERENCES}

1. K. M. Davis, K. Miura, N. Sugimoto, and K. Hirao, Opt. Lett. 21, 1729 (1996).

2. J. Zhang, M. Gecevičius, M. Beresna, and P. G. Kazansky, Phys. Rev. Lett. 112, 033901 (2014).

3. J. Cao, B. Poumellec, F. Brisset, A.-L. Helbert, and M. Lancry, J. Opt. Soc. Am. B 33, 741 (2016).

4. L. Sudrie, M. Franco, B. Prade, and A. Mysyrowicz, Opt. Commun. 171, 279 (1999)

5. H. Rong, R. Jones, A. Liu, O. Cohen, D. Hak, A. Fang, and M. Paniccia, Nature 433, 725 (2005).

6. G. Meltz, W. W. Morey, and W. H. Glenn, Opt. Lett. 14, 823 (1989).

7. E. V. Zavedeev, V. V. Kononenko, and V. I. Konov, Laser Phys. 26, 016101 (2015).

8. M. Chanal, V. Yu. Fedorov, M. Chambonneau, R. Clady, S. Tzortzakis, and D. Grojo, Nat. Commun. 8, 773 (2017).

9. I. Pavlov, O. Tokel, S. Pavlova, V. Kadan, G. Makey, A. Turnali, Ö. Yavuz, and F. Ö. Ilday, Opt. Lett. 42, 3028 (2017).

10. M. Chambonneau, Q. Li, M. Chanal, N. Sanner, and D. Grojo, Opt. Lett. 41, 4875 (2016).

11. O. Tokel, A. Turnalı, G. Makey, P. Elahi, T. Çolakoğlu, E. Ergeçen, Ö. Yavuz, R. Hübner, M. Z. Borra, I. Pavlov, A. Bek, R. Turan, D. K. Kesim, S. Tozburun, S. Ilday, and F. Ö. Ilday, Nat. Photonics 11, 639 (2017).

12. G. Matthäus, H. Kämmer, K. A. Lammers, C. Vetter, W. Watanabe, and S. Nolte, Opt. Express 26, 24089 (2018).

13. P. C. Verburg, L. A. Smillies, G. R. B. E. Römer, B. Haberl, J. E. Bradby, J. S. Williams, and A. J. Huis in 't Veld, Appl. Phys. A 120, 683 (2015).

14. Q. Li, M. Chambonneau, M. Chanal, and D. Grojo, Appl. Opt. 55, 9577 (2016).

15. H. Iwata, D. Kawaguchi, and H. Saka, Microscopy 66, 328 (2017).

16. H. Iwata, D. Kawaguchi, and H. Saka, Microscopy 67, 30 (2018).

17. M. Born and E. Wolf, Principles of Optics, 4th ed. (Pergamon, 1970), Chap. VIII.

18. M. Moharam and T. Gaylord, J. Opt. Soc. Am. 71, 811 (1981).

19. J. W. Goodman, An Introduction to Fourier Optics (McGraw-Hill, 1968). 